\title{
Interactive Performance Art Using Musical Instrument Daegeum for Healing
}

\author{
YoungMi Kim ${ }^{\star}$ \\ Singyoung-dong, Jongro-gu, 110-830, Seoul, South Korea \\ ymkimlab@naver.com
}

\begin{abstract}
The piece called healing bamboo is a drawing concept utilizing daegeum interface as a performance interactive art. The piece has an aim of manifesting spiritual culture of Bamboo healing by drawing fake bamboo on screen while daegeum is being played. Drawing bamboo trees was a representative healing method of the Korean ancestors. Although joys and sorrows of our ancestors differ from those of modern day, the idea of bamboo tree purifying consciousness and making mind upright continued up until now. A performer draws pictures by meditating upon the symbolization of bamboo trees and the old classical scholar' spirits. Accordingly, the daegeum sound that embraces mental values of an oriental culture and the bamboo tree drawing based on such sound offer emotional elements to heal the mind and body of a performer. We reinterpreted the cultivation of mind of bamboo tree healing as a modern tendency walking with the trend without discoloration of its meaning.
\end{abstract}

Keywords: Healing Art, Interactive Art, Performance, Cultural Technology.

\section{Background}

With economical advancements, modern people take one step further from just being rich and show high interests in managing healthy lives by healing mind and soul. The healing fever is the reflection of our lives being weary and hard. Because of that, it is necessary to first examine the cause before healing wounds. The emergence of healing appears to be attributable to stress from prolonged recession and demand to cure wounds from interpersonal relationships. Due to such reasons, healing is a new trend culture in the modern world. Art heals wounds in one's mind and soul and by that we can gain stability and pleasure. In most cases healing occurred through drawings and listening to music; however in this paper, we suggest art healing from the perspective of experience. Notably, in the past years, our ancestors recovered and healed wounded self-regard through art and music. In this paper, we focused on the Korean conventional culture forming the base of an oriental culture for consideration.

\footnotetext{
* Please note that the LNCS Editorial assumes that all authors have used the western naming convention, with given names preceding surnames. This determines the structure of the names in the running heads and the author index.
} 


\section{Bamboo Trees with Ideology of Healing}

Oriental esthetics is mostly embedded with the idea of mental healing. Interestingly, our oriental ancestors said we can disciple one's mind by just looking at an object which has mental values. As historically investigated, the representative plant with mental values is a bamboo tree and classical scholars observed bamboo trees by day and night and developed their mental, by drawing Bamboo trees for healing. This has values of drawing inner side and mind of the painter, not just copying the appearances of the bamboo trees in reality. They assumed the artistic activity of drawing bamboo trees was a way of mind culturing, incorporating philosophy, literary value, moral and cultivation.[1] Bamboo trees were perceived as an emblem of a man of virtue by our ancestors. [2] The symbolization of a bamboo tree as a man of virtue is due to five reasons which embrace powerful virtues.

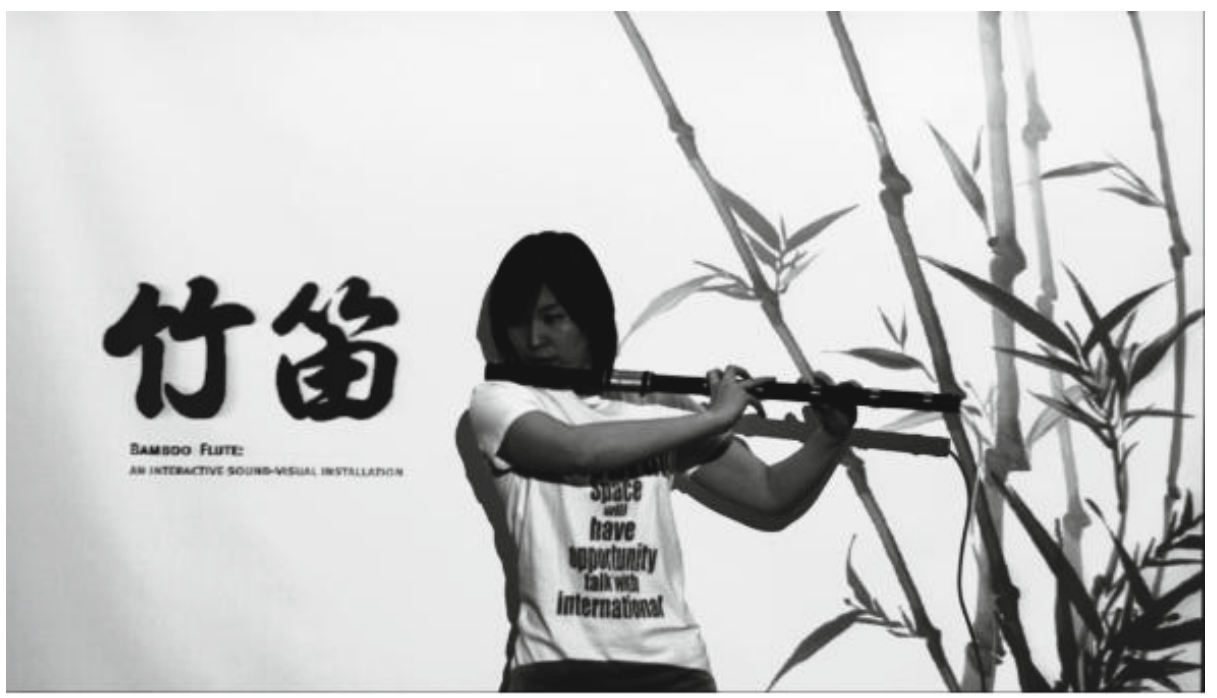

Fig. 1. The artwork as it is drawn according to a performers play

First is that bamboo tree has firm roots. A benevolent person should emulate bamboo roots and nurture virtues not to be pulled off.

Second is that bamboo tree has straight stem. We should not tilt to one side by straightening our mind.

Third is that bamboo trees are hollow inside. We should learn and cultivate ourselves with open mind, accepting others with open-mindednessor in other words, modestheart.

Fourth is that bamboo tree has straightened joints and has discipline. We should cultivate behaviors through straightness. 
Fifth is that bamboo tree never withers, being green for four seasons. It has unvarying mind in any circumstances.

Our forbearers printed the five characteristics of bamboo trees in their minds and drew bamboo trees keeping in their mind spirit. The mind of a bamboo tree is the uprightness, maintaining center and taking a firm stand in the fierce wind (harsh adversity).[3] We can see from bamboo drawings the wisdom of our forbearers that we can learn to stand upright whilewavering in the wind.

\section{Development of Bamboo Trees as an Interactive Art}

\subsection{Art Processing}

For <The healing bamboo> piece, we referred to the paintingby Leejung who established pattern tradition of bamboo tree painting in Chinese ink in Chosun dynasty.The Joseon Dynasty of Korea lasted more than 519 years and encompassed the reigns of 27 kings. During this time, which ink-and-wash painting flourished and became highly developed. Leejung's bamboo drawing in chinese ink represents bamboo drawings of Chosun dynasty in chinese ink by taking and developing one's line completely that you can not find earlier styles of bamboo
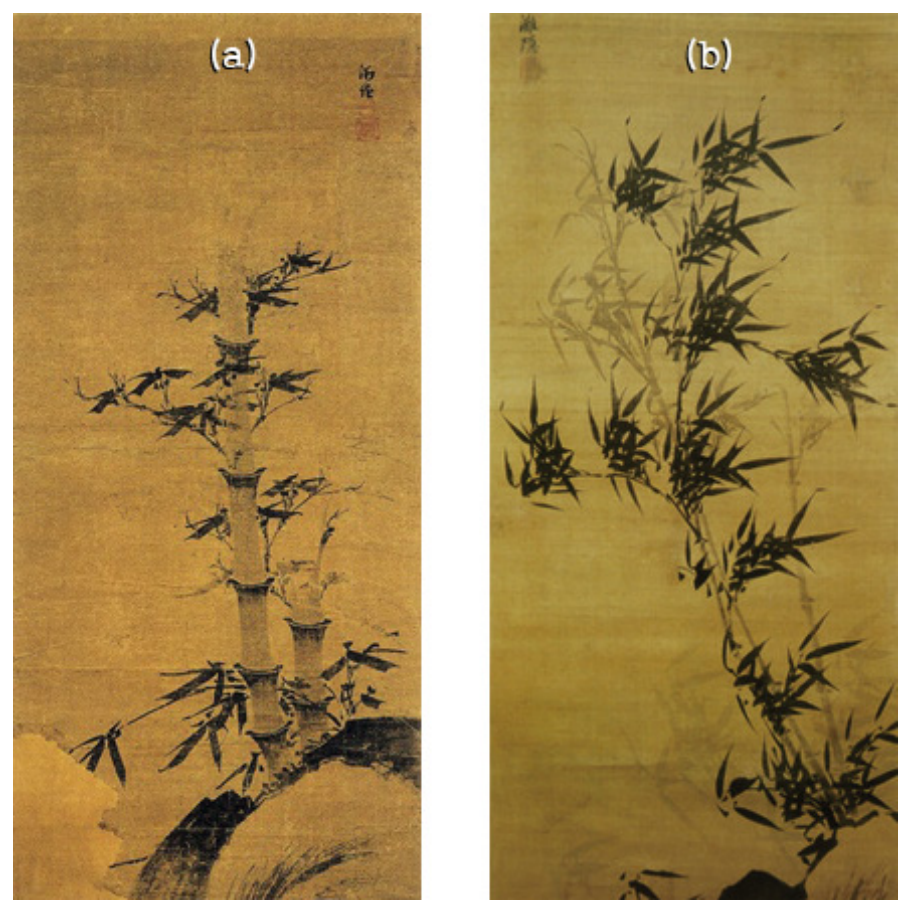

Fig. 2. Lee jung by bamboo trees (a)bamboo tree painting in chinese ink, Lee jung (b) wind-bamboo drawing, in the first half of 17 th century, Lee jung 
drawing in chinese ink in his drawings. (see Fig. 2) We created dual version, separating the old paintings of bamboo with thick nodes and wind-bamboo drawing which are Lee Jung's representative pieces. For the first version, we made references to (a)bamboo drawing in chinese ink and for the second version, we designed with references to (b)wind-bamboo drawing.

This drawing portrays the fidelity of classical scholars hardening firm will to live, recovering from hardships by bamboo trees. By the drawing (a) (see Fig. 2) showing bamboo trees which are upright, full of vigor, Leejung cherishes fidelity and integrity that classical scholars should have. (b) In this painting, Leejung focused on expressing condensed spirits of bamboo trees withstanding wind. Although the stem is straightened, the wisdom that we should deal with everything flexibly is passed on to other generations by the portrayal of a stem which is little bit bent. Because of these reasons, <wind-bamboo drawing>is illustrated in the Korean bill. Wind-bamboo drawing by Lee jung is used as a background picture for the current Korean 50, 000 won bill. The attitude of the old classical scholars drawing four gracious plant to cultivate minds and appreciating the drawings to become a man of virtue is much needed in this contemporary world. In the Fig. 3(a) we added modern touch in expressing bamboo with thick nodes in Leejung's Fig. 2(a). With strong drawing line, we added strength in each of the leaves one by one. Heavy breathing is expressed by drawing line of heavy concentration and drawing line of relatively weak breathing is weakened to give a three-dimensional effect by having a feeling of space.
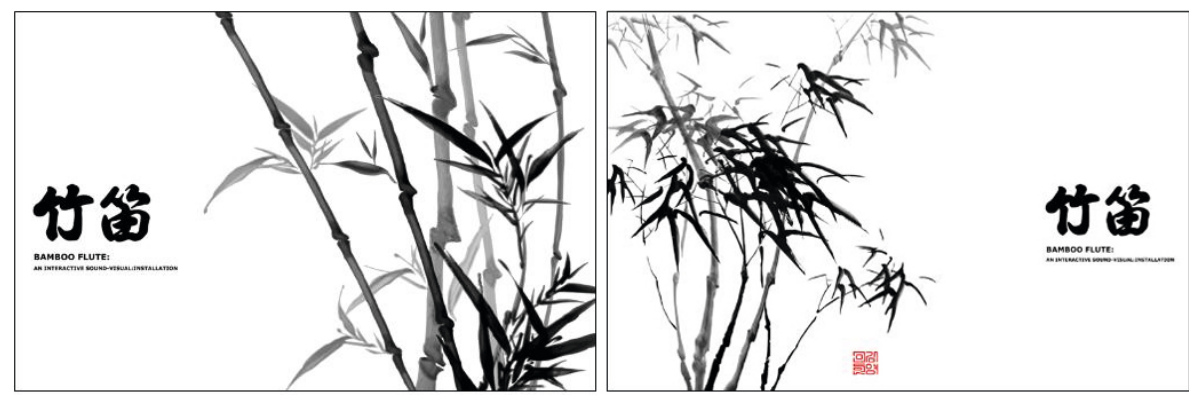

Fig. 3. Healing bamboo (a) "version 1" digital data with a reference to Fig. 2(a) a image (b) "version 2" digital data with a reference to Fig. 2(b) image

In the Fig. 3(b) elasticity of the bamboo is well-illustrated by accurately capturing bamboo stem and leaves fluttering in the wind. The sense of fluttering is well-portrayed as the bamboo stem is thin with thick leaves. This aims to nurture the mind to flexibly deal with an unexpected headwind in the modern life by drawing the bamboo confronting the wind flexibly without shaking in the coming wind. The completed bamboo tree slowly wavers by the wind by 15 degrees left and right and maintains dynamic movement until the seal is marked. The Fig. 3(b) adds dynamic interactive elements by improvement of what the Fig. 3(a) lacked which is made one year earlier than the Fig. 3(b). 


\subsection{Breathing as an Interactive Impersonation Tool}

The conventional four gracious plants were drawn by brush and ink. However in this piece, a daegeum as an interface replaces the formative meanings brush and ink took. The daegeum was an musical instrument which healed our ancestors in the past. [4] The daegeum makes very deep and pure sound by resonance and the tone seemed to represent and express feeling of lamentation and pain of a man of virtue that it became the musical instrument of healing cherished by classical scholars.
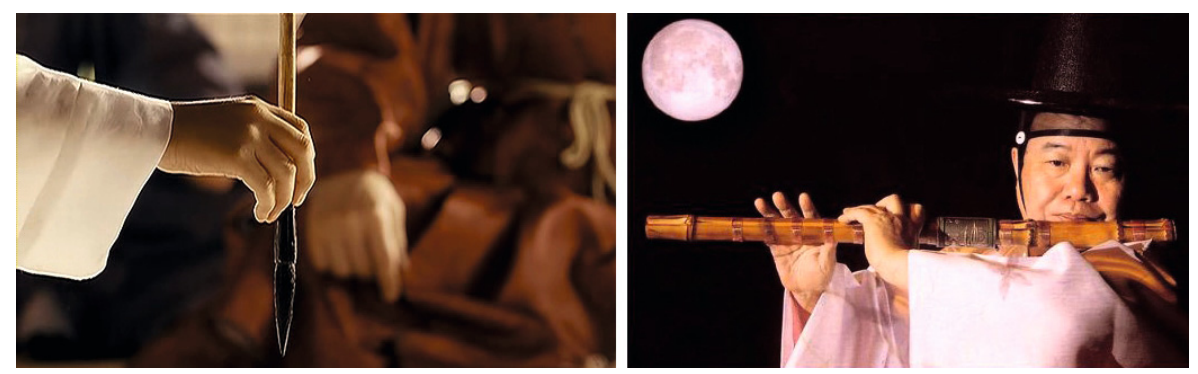

Fig. 4. The Interface (a)Drawn by brush and ink (b)Daegum: Daegum is one of the representative musical instruments that were played in palaces during Chosun Dynasty

$<$ The healing bamboo> is an art embodied in breathing. For the morning breathing method, which is the representative of an oriental mental cultivation method, we must exhale long and thin breaths through mouth, visualizingthe breath spreads out to every inch of the entire body to remove distracting thoughts. Prior to the exhaling, we need an inhaling procedure, in which we breath in heavily by nose to keep equilibrium. Such breathing procedures manipulate consciousness and removes distracting thoughts from head and maintains a state of equilibrium, leading to mind healing. The bamboo drawing made by calm and consistent breathing through daegeum maintains consistency in light and shade of ink stick. To copy bamboo in heart onto a screen, it is important to find relaxed and untrouble breathing. By this process, modern man can read the heart of the classical scholars which mimics the heart of a man of virtue and can heal mind and soul naturally.

Such breathing procedures manipulate consciousness and removes distracting thoughts from head and maintains a state of equilibrium, leading to mind healing. The bamboo drawing made by calm and consistent breathing through daegeum maintains consistency in light and shade of ink stick. To copy bamboo in heart onto a screen, it is important to find relaxed and untrouble breathing. By this process, modern man can read the heart of the classical scholars which mimics the heart of a man of virtue and can heal mind and soul naturally. 


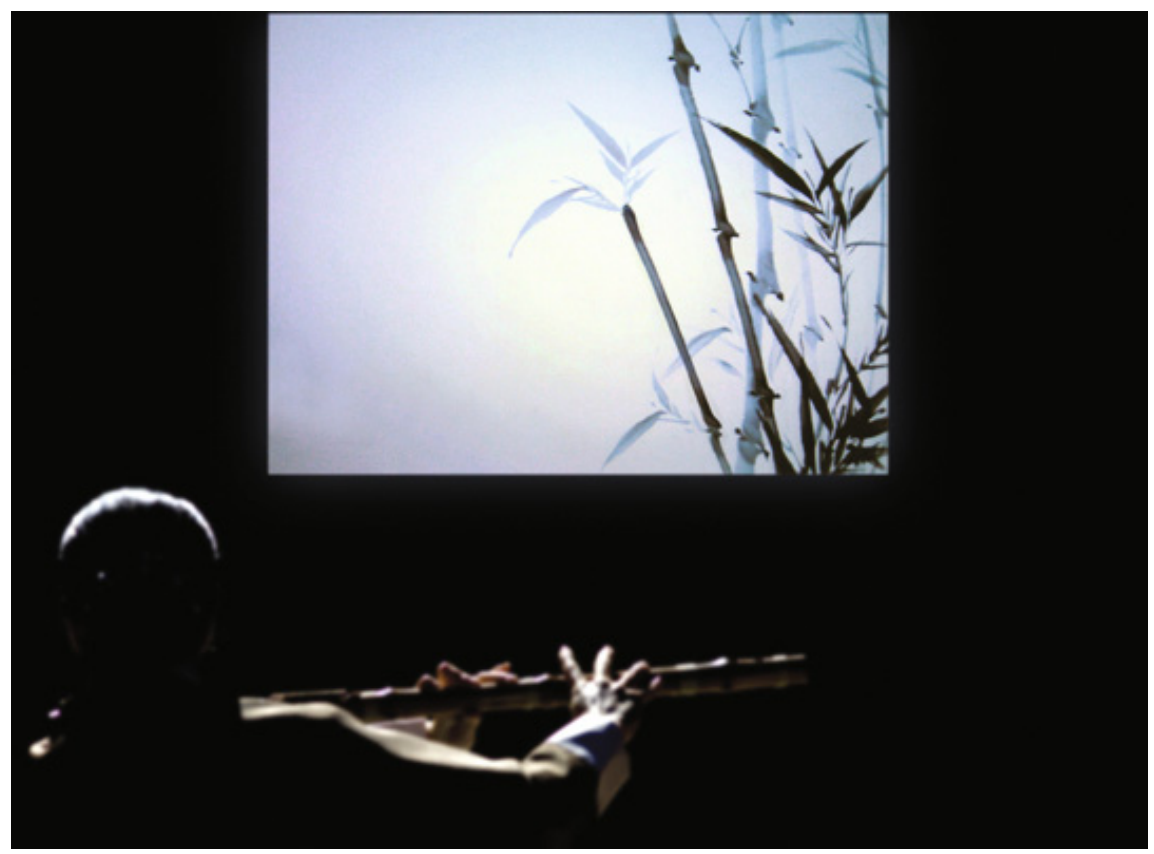

Fig. 5. Interactive Media Art for Healing exhibition, 2009

\subsection{Appropriate Technology Design}

The user sounds amplitude is the higher, the drawing line is the thicker. The others, the user sounds time is the longer, the drawing line is the longer. Finally, we can display the bamboos steps like black-and-white drawing on the screen using the drawing action quantized by decibel descriptor.[5][6]

Manufactured program, which digitalized analog features, is Adobe Flash program with a huge merit of establishing strong controller, which only the writers could use on. In the Figure 6, we will examine control panel in the upper side from left to right one by one. In the main menu in the left, the sound level of microphone level is at maximum 100. It becomes sensitive as the figure is lowered and will insensitively accept input values as the figure gets higher.

Blow Accumulation is a menu amassing breathing and a special daegeum sound is output when breathing strengths accumulate above a set up value. The accumulated numbers are not shown by figures but are shown by the expression of a gauge bar filled with grey color in graphics. The submenu, Processed Brush Power refers to the concentration when we draw bamboo trees. With a maximum strength of 110, heavier concentration on joints and leaves are portrayed as the figure gets higher. Process Brush Power inputs graphics and are classified by five stages $0-20,21-40,41-60,61-80,81-110$. Sensitivity refers to the drawing speed and bamboo joints are drawn quicker as the figure approaches 50 which is the maximum number. 


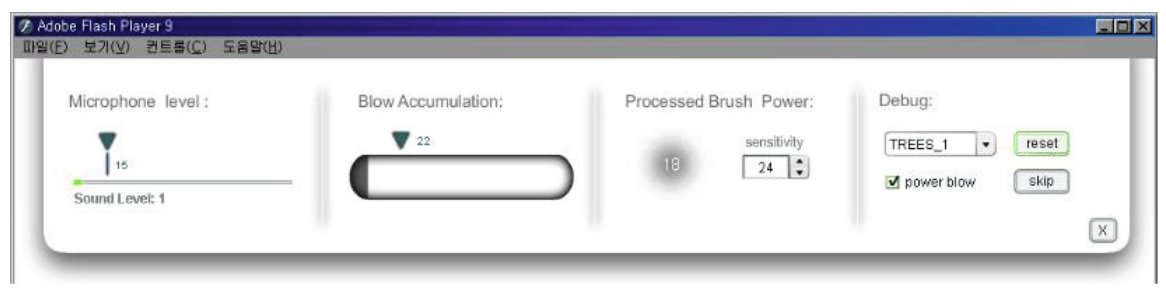

Fig. 6. Display controller

\section{Conclusion}

Real healing should expand consciousness and purify minds beyond healing body and heart. When we consider that mental crisis of our society is due to the lost meaning of life and the lack of self-control abilities, the necessity of mental cultivation is clearly noted. Our ancestors said a tranquility of mind and a mental healing occurs by just looking at an object of mental values. <The healing bamboo> expresses mental values and ideological meanings possessed by the subject well, through embodiment of gallery. The daegeum sound which mimics clear minds of the classical scholars and the heavy brush touch emotionally approached the mental cultivation of modern men. Consequently, through several exhibition, the approachment of the art as the interactive art seeking healing was positively valued.

\section{References}

1. Kim, Y.-M., Choi, J.-S.: Breathe Brush. In: 37th ACMSIGGRAPH 2010, Los Angeles, July 25-29 (2010)

2. Kim, Y.-M., Choi, J.-S.: Bamboo Flute. In: ACE 2009, October 29-30 (2009)

3. Choi, B.-S.: Thought and History of Ink Painting, p. 23. Dongmoonsun, Korea (2008)

4. Kim, Y.-M.: Interactive Ink-and-Wash Drawing. In: Anacleto, J., Fels, S., Graham, N., Kapralos, B., Saif El-Nasr, M., Stanley, K. (eds.) ICEC 2011. LNCS, vol. 6972, pp. 383-386. Springer, Heidelberg (2011)

5. Choi, B.-S.: Thought and History of Ink Painting, pp. 83-88. Dongmoonsun, Korea (2008)

6. Kim, Y.-M., Choi, J.-S.: The Sound Brush Made of Bamboo. In: Butz, A., Fisher, B., Christie, M., Krüger, A., Olivier, P., Therón, R. (eds.) SG 2009. LNCS, vol. 5531, pp. 279-282. Springer, Heidelberg (2009) 